\title{
Structuring temporal sequences: Comparison of models and factors of complexity
}

\author{
PETER ESSENS \\ TNO Human Factors Research Institute, Soesterberg, The Netherlands
}

\begin{abstract}
Two stages for structuring tone sequences have been distinguished by Povel and Essens (1985). In the first, a mental clock segments a sequence into equal time units (clock model); in the second, intervals are specified in terms of subdivisions of these units. The present findings support the clock model in that it predicts human performance better than three other algorithmic models. Two further experiments in which clock and subdivision characteristics were varied did not support the hypothesized effect of the nature of the subdivisions on complexity. A model focusing on the variations in the beat-anchored envelopes of the tone clusters was proposed. Errors in reproduction suggest a dual-code representation comprising temporal and figural characteristics. The temporal part of the representation is based on the clock model but specifies, in addition, the metric of the level below the clock. The beat-tone-cluster envelope concept was proposed to specify the figural part.
\end{abstract}

The ability of humans to capture temporal relationships among events plays a crucial role in the processing of information. Characteristics of this process have been studied in the perception of temporal sequences, sequences of tones in which tone onset intervals are varied. Two structures have been proposed for the representation of temporal sequences: time categories (Fraisse, 1946, 1956) and higher order time intervals (Jones, 1976; Lee, 1985; Longuet-Higgins \& Lee, 1982, 1984; Martin, 1972; Povel, 1981; Povel \& Essens, 1985). Support for these structures is found in experimental evidence that certain temporal relationships can be reproduced more accurately than others. They provide a structural basis for schemes that guide attentional processes to critical information in the world (Jones \& Yee, 1993). For instance, pitch recognition is better for tones that mark the higher order time intervals (Monahan, Kendall, \& Carterette, 1987).

Fraisse $(1946,1956)$ found that time intervals that had a 2:1 relationship were more accurately reproduced than others. Fraisse concluded that a representational structure exists that has two categories, long and short, related as $2: 1$, onto which time intervals are mapped. Povel (1981) found empirical evidence for higher order time structures. He found that sequences with interval relations of $3: 1$ and $4: 1$ were represented adequately if these intervals were embedded in a higher order structure that fit the se-

The author gratefully acknowledges Carol A. McCann for extensive comments and discussion; Jeroen G. W. Raaijmakers, John A. Michon, Stephen Handel, Caroline Monahan, Lester Krueger, and DirkJan Povel for reviewing a draft of this manuscript; Tom Vijlbrief and Evert Agterhuis for software and hardware support. Requests for reprints should be sent to P. Essens, TNO Human Factors Research Institute, P.O. Box 23, 3769 ZG Soesterberg, The Netherlands (e-mail: petere@tm.tno.nl).

-Accepted by previous editor, Charles W. Eriksen quence. This higher order structure can be represented as a time scale consisting of recurrent time units that encompass the (shorter) intervals in the sequence. Povel called this hierarchical structure a beat-based structure, with the beats marking the onset points of the consecutive time units of the time scale. Essens and Povel (1985) compared the accuracy of reproduction of sequences that were embedded in a hierarchical structure with the same sequence presented alone. The former were reproduced better than the latter, with the exception of cases in which there was a 2:1 relationship in the intervals in the sequence. In those cases, reproductions were equal in quality. This suggests that a $2: 1$ representation is a basic scheme that is adopted if no higher order structure can be found. Indeed, the sequences that Fraisse used did not allow for hierarchical structuring.

Povel and Essens (1985) proposed two models that address different aspects of the coding of a temporal sequence. According to Povel and Essens, the coding of a sequence consists, first, of the selection of a higher order time scale as a metric to segment the sequence into equal time units, and subsequently, of the specification of the intervals in the sequence in terms of subdivisions of the higher order time units. The first stage is addressed by the clock model, which is a further, algorithmic, development of the beat-based concept. The clock model predicts which of many possible time scales (clocks) is the best for structuring a particular sequence and specifies how good the scale is. Sequences differ in how good their best time scale is. The better a sequence can be metrically structured, the better it will be represented and reproduced. For the second stage in the coding of temporal sequences, Povel and Essens formulated the subdivision model. ${ }^{1}$ The model distinguishes different kinds of subdivisions (equal and unequal) and asserts that unequal subdivisions are more complex to code than equal ones. Both the metrical structure and type of sub- 
division are assumed to contribute to the complexity of a sequence.

The two-stage approach that bases the organization of a temporal sequence on a higher level time unit differs from proposals in which the smallest interval in the sequence is used to build a hierarchy (Longuet-Higgins \& Lee, 1984; Martin, 1972). However, Essens and Povel (1985) found no support for the hypothesis that the smallest interval is used as a basis for structuring the sequence. Longuet-Higgins and Lee (1982) and Lee (1985) developed process models that address how people build a hierarchical structure while processing a sequence. They proposed that the first long interval in the sequence is used as a tentative higher order interval on which a metrical structure can be based. The stress on using information from the beginning of the sequence results in metrical predictions that differ from those of the clock model. The algorithmic nature of these models (Essens \& Povel, 1985; Lee, 1985; Longuet-Higgins \& Lee, 1982, 1984) allowed for a precise assessment and comparison of the models, which is described in the first part of this paper (Experiment 1). The second part focuses on the relationship between the clock selection and subdivision and the extent to which these factors account for the complexity of the sequence. The degree to which these two factors contribute to the complexity of a sequence is assessed in Experiments 2 and 3.

\section{PART 1 \\ Comparison of Models}

\section{The Clock Model of Povel and Essens (1985)}

The model assumes that listeners use a mental clock as a time scale to structure a sequence that is presented to them. For example, consider the following sequence with tone onset intervals of $200,400,400,200,200$, 400 , and $600 \mathrm{msec}$ (in units of $1: 1221123$ ), which is represented on a time axis in Table 1. Vertical lines indicate tone onsets. The duration of the tone is not relevant here, and is set to $50 \mathrm{msec}$ in the following experiments. The distance between two lines represents visually the size of an interval between two tone onsets. The dots help one to see the relative time (a dot marks how the smaller interval fits in the larger). At least in theory, many different matching time scales (clocks) can be conceived, varying in unit size and in their synchronization with the sequence. Each clock groups the intervals in the sequence differently, resulting in different representations of the sequence. However, the only viable clocks are those that are divisors of the sequence. The sample sequence, having a length of 12 , thus has no clock of unit 5 . The list of clocks given in Table 1 might suggest that listeners develop an unlimited number of higher order representations for this sequence. However, if one plays out sequences like this one in a cyclical way, considerable agreement can be found across listeners on the preferred structure (sometimes called the "natural" interpretation). The clock model addresses the factors that deter-
Table 1

Potential Time Scales (Clocks a-i) for Sequence 1221123 With Calculated Counterevidence

\begin{tabular}{|c|c|c|c|c|c|}
\hline \multirow[b]{3}{*}{ Clock } & Sequence & \multirow{3}{*}{$\begin{array}{c}\text { Clock } \\
\text { Size }\end{array}$} & \multirow{2}{*}{\multicolumn{3}{|c|}{ Counterevidence }} \\
\hline & $>>>>>$ & & & & \\
\hline & $|1.| .|| 1 . \mid \ldots !$ & & Oev & $-\mathrm{ev}$ & $\mathrm{C}^{*}$ \\
\hline a & $i . i . i . i . i . i$. & 2 & 2 & 4 & 18 \\
\hline$b$ & .i.i.i.i.i.i & 2 & & 1 & 4 \\
\hline $\mathrm{c}$ & $i \ldots i \ldots i \ldots i \ldots$ & 3 & 2 & & 2 \\
\hline $\mathrm{d}$ & i..i. . . i. & 3 & & 2 & 8 \\
\hline $\mathrm{e}$ & . i. i..i.i & 3 & & 3 & 12 \\
\hline f & $i \ldots i \ldots i \ldots$ & 4 & 1 & 2 & 9 \\
\hline $\mathrm{g}$ & i...i... & 4 & & & 0 \\
\hline $\mathrm{h}$ & $\ldots i \ldots i \ldots i$ & 4 & 1 & 2 & 9 \\
\hline
\end{tabular}

Note-1 = tone onset that marks interval; smallest interval is $200 \mathrm{msec}$; dots indicate relative time; $>$ marks tone perceived as accented; ! = first tone next cycle; $i=$ beat. ${ }^{*} \mathrm{C}$-score $=0 \mathrm{ev}+(4 *$ -ev).

mine the selection of the clock and predicts which clock is preferred.

Two factors are critical in selecting the preferred ("best") clock to structure the sequence: the perception of accented tones in the sequence, and the quality of match between a potential clock and the sequence of accents perceived in the sequence. Accented tones are those that are perceived by human listeners as more salient than others. This is certainly the case for tones that deviate in loudness, length, or pitch. In addition, it has been found that even in sequences of identical tones, differences in salience are perceived between the tones, depending on the relative size of the time intervals between them. This led to the following accent rule: Tones that are preceded or followed by relatively long tone onset time intervals are perceived as more salient than others, except for the first of a cluster of two tones. In two-tone clusters, the second tone is perceived as more accented than the first (Povel \& Okkerman, 1981). In the sample sequence in Table 1, only the first two tones of the (cyclically presented) sequence form a two-tone cluster, because the two are separated from the other tones by a longer interval. The third tone is single; the fourth, fifth, and sixth tones form a three-tone cluster; the seventh is single. According to the accent rule, the second, third, fourth, sixth, and seventh tones will be perceived as accented (indicated by " $>$ ").

The clock model proposes that the clock selected to structure the sequence will be the one that matches the sequence of accents best. A perfectly matching clock is a clock with all clock ticks (beats) coinciding with accented tones. Mismatch of beats and accents is expressed as counterevidence against a clock. Two cases of counterevidence are distinguished: one where a beat coincides with a tone that is not accented (termed $0 \mathrm{ev}$ for zero evidence); and one where a beat does not coincide with a tone at all (termed $-\mathrm{ev}$ for negative evidence). The total amount of counterevidence (C) against a given clock is expressed as a $\mathrm{C}$-score, and is calculated as the sum of 
the number of instances of $0 \mathrm{ev}$ and - ev, the latter multiplied by a weighting factor $(\mathrm{W})$. The weighting factor adjusts for the relationship between $0 \mathrm{ev}$ and $-\mathrm{ev}$, since the latter is considered to give stronger counterevidence than the former ( $\mathrm{W}$ is set to 4 in this paper). ${ }^{2}$ The algorithm computes the counterevidence for all clocks of the sequence and selects the clock with the lowest C-score as the "best clock." The clock with the lowest C-score will have the highest probability of being selected for structuring the sequence.

In Table 1, a number of theoretically possible clocks are shown for the sequence 1221123. Clock $a$ with size two has two clock beats (Beats 1,4$)$ that coincide with unaccented tones (Tones 1,5 ) and four that have no matching tones, which results in two 0ev's and four - ev's, respectively. The C-score [0ev $+(4 *-\mathrm{ev})]$ equals $2+(4 * 4)=18$.

An issue for the model is to decide on the upper boundary of the clock unit that should be considered. The largest clock unit size for a sequence is the one equal to the length of the sequence itself. Such a clock may have little counterevidence, but it does not seem to be efficient in grouping the intervals of the sequence. The same can be said for clocks that have a unit length half that of the sequence (here, length 6). Although there is as yet no firm theoretical basis for setting the limit, Povel and Essens (1985) assumed that only clocks that were less than half the length of the sequence were efficient in organizing the sequence. The $\mathrm{C}$-scores can be used to organize sequences into categories on the basis of the amount of counterevidence associated with the best clock for each sequence. For some sequences, the algorithm might find clocks with equal $\mathrm{C}$-scores. These clocks have an equal chance of being selected as the clock for structuring the sequence.

Sequences that cannot be subdivided into equal time units (e.g., a sequence of length 13) have no valid clocks and have to be structured in other ways. These sequences were termed nonmetrical. Sequences for which valid clocks can be defined are called metrical. The clock unit is referred to as the metrical unit. Notice that the clock model does not specify the metric of the intervals smaller than the clock unit. The temporal structure of these is specified by the subdivision model.

If we assume that a listener will try to find a mental clock to structure a sequence and will adopt the one with the least counterevidence (the "best clock"), two predictions can be tested: (1) a sequence will be reproduced more accurately if it can be perceived against a mental clock; (2) a sequence having a best clock with a C-score that is lower than that of another sequence will be reproduced more accurately. The first prediction was tested by Essens and Povel (1985). They found that inaccuracy of reproduction was greater in nonmetrical sequences than when the same sequences were embedded in larger metrical sequences. Povel and Essens tested the second prediction. They compared the accuracy of reproduction of sequences which differed in the $\mathrm{C}$-score of their best clock. The results supported the prediction that the $\mathrm{C}$-score is indicative of how well a sequence is structured. These experiments demonstrate that the mental clock concept is a valid and powerful concept.

\section{The Models of Longuet-Higgins and Lee $(1982,1984)$}

Longuet-Higgins and Lee (1982) described a model that predicts which higher order temporal structure will be established by the listener. The model assumes that in the process of listening, the listener develops hypotheses about the structure of the sequence. The model is a process model, in the sense that it operates from left to right through a sequence. A major assumption is that tones that start relatively long intervals initiate major metrical units. ${ }^{3}$ Another assumption is that metrical units are combined going up the metrical hierarchy. Lee (1985) refined the model of Longuet-Higgins and Lee (1982) concerning the building of the hierarchy of metrical levels. We will discuss the effect of initial long intervals in more detail when addressing Lee (1985).

Longuet-Higgins and Lee (1984) presented another model of the metrical interpretation of sequences that assumes that listeners tend to avoid an interpretation that results in syncopations. Only "natural interpretations"that is, those in which the beats coincide with toneswill be selected. In contrast to the earlier process model, this model considers all intervals in a sequence in defining the metrical units. The algorithm looks first for the shortest interval; this defines the smallest metrical unit. The second step is to consider the distances (higher order intervals) between the tones that are the onsets of intervals longer than the established metrical unit. If these higher order intervals are multiples of an already established metrical unit, the value of the new, higher order, metrical unit is set to the lowest common divisor of the higher order intervals, or, if there is only one kind of higher order interval, to the size of the higher order interval itself. Consider the sequence with relative interval sizes $4,1,1,4,1,1,2,1,2,1,3,3$ (from their paper). The spacing of the onsets of the longer intervals (longer than 1$)$ is $663333(4,1,1 ; 4,1,1 ; 2,1 ; 2,1 ; 3 ; 3)$. The common divisor is 3 , so the size of the metrical unit is 3 . There are two intervals longer than 3 with their tone onsets spaced by 6 . This is sufficient to establish a metrical unit of size 6 . The metrical analysis of the sequence results in a two-level hierarchy of metrical intervals of sizes 3 and 6 , respectively.

The model is limited because it can only find metrical units (greater than one) for sequences that have a regular spacing of the long intervals. What is the predictive capability of the Longuet-Higgins and Lee (1984) model? An implementation of the model as a computer algorithm was used to assess its capabilities quantitatively. The approach used in our research involved the generation of large sets of sequences consisting of noncyclical permutations of intervals with unit lengths 4,3 , 2 , or 1 with total lengths of 12 and 16 . These sets, spec- 
Table 2

Number of Valid Interpretations Relative to the Total Number of Sequences (in Percent) With Lengths 12 (Set12) and 16 (Set16)

\begin{tabular}{lcc}
\hline \multicolumn{1}{c}{ Models } & $\begin{array}{c}\text { Set12 } \\
(n=329)\end{array}$ & $\begin{array}{c}\text { Set16 } \\
(n=3,189)\end{array}$ \\
\hline Povel \& Essens, 1985 (P\&E) & 100 & 100 \\
Longuet-Higgins \& Lee, 1984 & 39 & 15 \\
Lee, 1985 & 90 & 98 \\
Same predictions as P\&E & 29 & 12 \\
Different from P\&E & 61 & 86 \\
\hline
\end{tabular}

ified as Set 12 and Set 16 and containing 329 and 3,189 different sequences, respectively, provided the sequences for testing the algorithmic models. The sets were analyzed by applying the algorithm, and subsequently, the number of metrical interpretations that the algorithm produced was counted. The number of interpretations was $128(39 \%)$ for Set 12 and $462(15 \%)$ for Set 16 (Table 2), a relatively small subset of the total number of sequences, compared with the coverage of the other models.

\section{The Model of Lee (1985)}

Lee (1985) questioned the strict requirement of the Longuet-Higgins and Lee (1984) model that the interpretation avoid syncopations. He argued that during the process of listening, the information in the beginning of the sequence could lead to an interpretation that forced a syncopation later in the sequence. Lee formulated an algorithmic model that incorporates the process model of Longuet-Higgins and Lee (1982). The algorithm, described in Table 3, uses the first interval in the sequence to tentatively define the size of the metrical unit (a first interval of size $=1$ is skipped, because it does not result in a meaningful metrical unit); subsequently it tests whether there is counterevidence against this hypothesis. Once a metrical level is established, it continues to search for a higher metrical level if possible.

The metrical structure predicted by Lee depends on the first interval encountered. If the sequence were presented starting on a different position, for example, a different structure might be found. Lee's model will not always result in a prediction for a metrical structure of a sequence. The strict requirement to accommodate longer intervals may result in continued adjustment until the end of the sequence is reached, with no conclusion. The model assumes that higher order metrical levels are built on the lower levels, which differs from the topdown approach taken by Povel and Essens (1985).

What is the predictive capability of the process model of Lee (1985)? We assessed this in the same way as for the model of Longuet-Higgins and Lee (1984). The numbers of valid interpretations produced by Lee's algorithm were $296(90 \%)$ and 3,135 (98\%) for Sets 12 and 16, respectively (see Table 2). It can be concluded that Lee's model is capable of finding metrical interpretations for a large range of sequences. The predicted metrical interpretations are, however, very different from those of

Table 3

Metrical Analysis of Tone Sequence According to Model of Lee (1985)

\begin{tabular}{|c|c|}
\hline & Sample Sequence* \\
\hline Analysis Steps & $|\ldots||\ldots \ldots| .|\cdot| \cdot \mid \ldots !$ \\
\hline $\begin{array}{l}\text { 1. Set size metrical unit }\left(t_{1}-t_{2}\right) \text { equal to length } \\
\text { of first interval }\end{array}$ & $\begin{array}{l}i \ldots i \\
t_{1}-t_{2}\end{array}$ \\
\hline $\begin{array}{l}\text { 2. Extrapolate } t_{1}-t_{2} \text { unit, such that } t_{1}-t_{2} \text { equals } \\
t_{2}-t_{3}\end{array}$ & $\begin{array}{l}i \ldots i \ldots i \\
t_{1}-t_{2}-t_{3}\end{array}$ \\
\hline $\begin{array}{l}\text { 3. IF there exists an interval } x \text { starting within } \\
t_{2}-t_{3} \text { greater than the interval starting at } t_{2}\end{array}$ & $i \ldots i \ldots i$ \\
\hline $\begin{array}{l}\text { THEN update } t_{1}-t_{2} \text { by moving } t_{2} \text { up to } \\
\text { interval } x \text {, GOTO Step } 2 \\
\text { ELSE a metrical level is established }\end{array}$ & $t_{1}--t_{2}--t_{3}$ \\
\hline $\begin{array}{l}\text { 4. IF there exists an interval } y \text { greater than size of } \\
\text { the metrical unit established AND the tone } \\
\text { that starts } y \text { is marked by a beat of a } \\
\text { previously established metrical level }\end{array}$ & $i \ldots i \ldots i$ \\
\hline $\begin{array}{l}\text { THEN set that tone to mark start }\left(t_{1}\right) \text { of the } \\
\text { higher metrical unit } \\
\text { ELSE stop }\end{array}$ & $t_{1}$ \\
\hline $\begin{array}{l}\text { 5. IF there is a next tone marked by a beat of the } \\
\text { previously established level }\end{array}$ & $\begin{array}{r}i \ldots i \ldots i \ldots i \\
i \ldots \ldots i\end{array}$ \\
\hline $\begin{array}{l}\text { THEN set that tone to } t_{2}{\text { (new } t_{1}-t_{2}} \\
\text { unit established) } \\
\text { ELSE stop }\end{array}$ & $t_{1}-\cdots---t_{2}$ \\
\hline $\begin{array}{l}\text { 6. Continue with Step } 2 \text { with condition that tones } \\
\text { should be matched by the previously } \\
\text { established beats }\end{array}$ & $\begin{aligned} & i \ldots i \ldots i \ldots i \ldots i \ldots i \\
& i \ldots \ldots i \ldots \ldots \text { i } \ldots t_{3}\end{aligned}$ \\
\hline
\end{tabular}

Note-“ “ $i$ " represents beats of a metrical level; " $t$," marks metrical units. * ${ }^{*}$ Lee (1985) represented sequences as note sequences; here a time axis notation is used. 
Table 4

Differences Between the Models of Povel and Essens (1985) and Lee (1985)

\begin{tabular}{|c|c|}
\hline Povel and Essens (1985) & Lee $(1985)$ \\
\hline $\begin{array}{l}\text { Information in the whole sequence } \\
\text { is used to determine interpretation. }\end{array}$ & $\begin{array}{l}\text { Information early in the sequence } \\
\text { determines interpretation. }\end{array}$ \\
\hline $\begin{array}{l}\text { Prominent tones are preferred onsets of } \\
\text { clock units; prominent tones (accents) } \\
\text { are tones followed by a long interval or } \\
\text { those preceded by a long interval } \\
\text { (except for two-tone groups). }\end{array}$ & $\begin{array}{l}\text { Tones followed by a long interval are } \\
\text { potential onsets of a metrical unit. }\end{array}$ \\
\hline $\begin{array}{l}\text { Counterevidence from the whole } \\
\text { sequence gives indication of adequacy } \\
\text { of a metrical hypothesis. }\end{array}$ & $\begin{array}{l}\text { Positive evidence from the first long } \\
\text { interval determines the initial } \\
\text { metrical hypothesis. }\end{array}$ \\
\hline $\begin{array}{l}\text { A metrical hypothesis that best matches } \\
\text { the accents is most likely to be selected } \\
\text { to interpret the sequence. }\end{array}$ & $\begin{array}{l}\text { A metrical hypothesis is established } \\
\text { once there is no counterevidence in } \\
\text { the second of the consecutive } \\
\text { metrical units. }\end{array}$ \\
\hline $\begin{array}{l}\text { Only one strict condition: unit size is a } \\
\text { divisor of the sequence length. }\end{array}$ & $\begin{array}{l}\text { There are several strict conditions; } \\
\text { therefore, a metrical hypothesis } \\
\text { cannot always be found. }\end{array}$ \\
\hline
\end{tabular}

Povel and Essens (1985). In a comparison of the two models, the numbers of equal predictions were counted. A prediction was scored as being the same if the best clock from Povel and Essens was equal to (one of) the metrical unit(s) predicted by Lee. Of Set12, 95 of the 296 solutions found by Lee's model were the same as for Povel and Essens's model. Comparisons in Set16 resulted in only 395 out of $3,135(13 \%)$ predictions being the same. The large discrepancy can be understood if we look at the units of the metrical structures predicted by Lee. Almost half of the 2,740 predictions in Set 16 that differed from Povel and Essens (1985) are inappropriate in terms of the Povel and Essens model because the predicted unit size is $3,5,6$, or 7 , none of which divides the sequence evenly. To summarize the comparison between Povel and Essens (1985) and Lee (1985), the distinctive features of the two models are presented in Table 4.

\section{EXPERIMENT 1}

The goal of Experiment 1 was to determine which of the two models is better at predicting the metrical structure used by listeners for structuring a temporal sequence. The fact that there are tone sequences in which the models predict different metrical structures (other than the nondivisor ones) makes it possible to test the adequacy of the two models. There is, however, no direct way to assess this. One method is to ask subjects to tap along with the sequence, the assumption being that the tapping reflects the metrical unit selected. A problem with this method is that subjects sometimes seem to lose the link between their tapping and the sequence, in particular for complex sequences. An alternative approach, also used by Povel and Essens (1985), is to add a lowpitch sequence of equal intervals (isochronic) to the test sequence, which induces a metrical structure. The induction effect can be so strong that the same test sequence combined with two different isochronic se- quences is heard as two different test sequences. This method was used here to compare the quality of the different metrical structures predicted by the two models. Sequences were selected from Set12, for which the two models predict a different metrical structure. Most of these were sequences for which Povel and Essens's model predicts a 4-clock as best clock, whereas Lee's model predicts a 3-clock. According to the clock model, the 3-clock is less adequate for the sequence, because it has a higher C-score than the 4-clock has. Only few sequences were found for which Lee's model predicted a 4-clock and Povel and Essens's a 3-clock.

\section{Method}

Subjects. Twelve subjects, members of the TNO Human Factors Research Institute, participated in the experiment. Five were musically trained, having played an instrument for at least 5 years.

Stimuli. From Set 12, 10 sequences were used for which the clock model predicted a different metrical structure than the Lee model. The sequences were selected on the basis of having low $\mathrm{C}$-scores. The sequences were combined with an isochronic sequence to induce the predicted metric. This resulted in two sets of stimuli: In the $P \& E$ condition, the metric predicted by the clock model of Povel and Essens (1985); in the Lee condition, the metric predicted by the model of Lee (1985). The test sequences are presented in Table 5 together with the way the predicted clock coincides with the tone sequence. In Table 5, Test Sequence 1 had tone onset intervals of $200,200,400,200,400,200,200$, and $600 \mathrm{msec}$ (in units of $1: 11212113$ ). The $\mathrm{C}$-score indicates the quality of the predicted clock according to the clock model. The stimuli were presented to the subjects as sequences consisting of tones of $50-\mathrm{msec} 830-\mathrm{Hz}$ square waves, with $3-\mathrm{msec}$ rise and fall times in combination with a low-pitch $(125-\mathrm{Hz}$, square waves) isochronic tone sequence that corresponded to the clock predicted by the model being tested. The beats of the isochronic sequence always coincided with tones in the sequence. Thus, there was never a low-pitch tone in an empty interval. The stimuli were presented cyclically, starting with the first beat-tone combination.

Procedure. The experiment was performed in two parts: first, one model condition, and after a short pause, the other condition. Order of conditions was balanced. Order of presentation of the stimuli within each condition was randomized. The task of the subject was to reproduce the presented test sequence as precisely 
Table 5

The Ten Sequences Used in Experiment 1 Combined With the Predicted Mental Clock

\begin{tabular}{|c|c|c|c|c|c|}
\hline \multirow[b]{2}{*}{ No. } & \multirow[b]{2}{*}{ Test Sequence } & \multicolumn{2}{|l|}{ Condition P\&E } & \multicolumn{2}{|l|}{ Condition Lee } \\
\hline & & Stimulus & $\mathrm{C}^{+}$ & Stimulus & $\mathrm{C}+$ \\
\hline 1 & 11212113 & $\begin{array}{l}|||\ldots||\cdot| \mid \ldots ! \\
.1 \ldots i \ldots i \ldots *\end{array}$ & 1 & 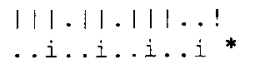 & 5 \\
\hline 2 & 11212122 & $\begin{array}{l}|||||.||\cdot| . \mid \\
i \ldots i \ldots i \ldots\end{array}$ & 1 & $\begin{array}{l}|||.||\cdot||.| . ! \\
\ldots i \ldots i \ldots i \ldots i\end{array}$ & 4 \\
\hline 3 & 1121214 & $\begin{array}{l}|||||||| \ldots ! \\
i \ldots i \ldots i \ldots\end{array}$ & 1 & $\begin{array}{l}|| \cdot||, \mid \ldots ! \\
\ldots i \ldots i \ldots i \ldots i\end{array}$ & 4 \\
\hline 4 & 1212114 & $\begin{array}{l}|| .|| \cdot|| \mid \ldots ! \\
i \ldots i \ldots i \ldots\end{array}$ & 1 & $\begin{array}{l}|| .|| \cdot|| \ldots ! \\
. i \ldots i \ldots i \ldots i .\end{array}$ & 5 \\
\hline 5 & 2121222 & $\begin{array}{l}|.||.||\cdot| . \mid \ldots ! \\
\ldots i \ldots i \ldots i .\end{array}$ & 1 & $\begin{array}{l}|\cdot| 1,|1 .| . \mid . ! \\
i \ldots i \ldots i \ldots i \ldots\end{array}$ & 4 \\
\hline 6 & 1121313 & $\begin{array}{l}|||||\ldots| \mid \ldots ! \\
. i \ldots i \ldots i \ldots\end{array}$ & 1 & $\begin{array}{l}|||||\ldots| \mid \ldots ! \\
\ldots i \ldots i \ldots i \ldots i\end{array}$ & 5 \\
\hline 7 & 1213113 & $\begin{array}{l}|| \ldots|| \ldots|| \mid \ldots ! \\
i \ldots i \ldots i \ldots\end{array}$ & 2 & 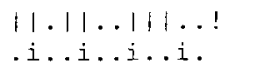 & 4 \\
\hline 8 & 121314 & $\begin{array}{l}||,|| \ldots|| \ldots ! \\
i \ldots i \ldots i \ldots\end{array}$ & 1 & 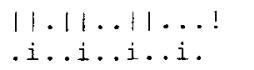 & 5 \\
\hline 9 & 111112122 & $\begin{array}{l}\|\|\|\ldots\| . \mid . ! \\
i \ldots i \ldots i \ldots\end{array}$ & 1 & $\begin{array}{l}||||||-|| .|.| \\
\ldots i \ldots i \ldots i \ldots i\end{array}$ & 5 \\
\hline 10 & 11121222 & $\begin{array}{l}|||1 .||.| . \mid . ! \\
\ldots i \ldots i \ldots i\end{array}$ & 1 & $\begin{array}{l}1111,11.1 .1 . ! \\
1 \ldots i \ldots i \ldots i \ldots\end{array}$ & 4 \\
\hline
\end{tabular}

Note-P\&E, Povel and Essens (1985); Lee, Lee (1985). Smallest tone onset interval is $200 \mathrm{msec}$. *For all 10 sequences: the predicted mental clock in the P\&E and Lee conditions. $\nmid C$-score.

as possible. The subjects listened to each stimulus and practiced tapping in synchrony with it as long as they wished. The subject stopped stimulus presentation by pushing a button, and then reproduced four cycles of the sequence on a response plate. Each tap produced the same tone as that used in the sequence. If the subject made a serious tapping error, resulting in a standard deviation that exceeded $15 \%$ of the mean of the tapped intervals, the subject was asked to produce that sequence again, repeating the procedure of listening and practicing the stimulus involved. Before the experiment started, the procedures were practiced with three training stimuli. Stimulus generation and response registration were controlled by an Apple Macintosh Plus computer. Tones were produced by sound generators. The low-pitch tones were controlled by a synchronized timer circuit that was triggered by a pulse from the computer.

\section{Results}

Accuracy of reproduction was expressed by the mean of the deviations between the intervals in the presented sequence and the reproduced sequence. The deviation score was calculated in the following way. First, for each sequence, the lengths of each of the corresponding intervals in the four cycles of the reproductions were averaged. For each interval of the sequence, the absolute difference between the actual length and the average reproduced length was calculated as a percentage of the actual length. Finally, these percentages were averaged over intervals. Examination of performance of the individuals did not yield differences related to musical training. The mean deviations (in percent) from Experiment 1 are presented in Figure 1, averaged over subjects for the 10 sequences in the two model conditions. Performance in the $P \& E$ condition is better overall than in the Lee condition $[F(1,11)=7.6, p<.05]$. Sequences differed from each other significantly $[F(9,99)=4.1$, $p<.01]$. This effect was particularly marked in the P\&E condition. There was, however, no interaction between conditions and sequences $[F(9,99)=1.8, p>.05]$.

\section{Discussion}

The analysis of the predictive capabilities of the three models examined indicates that the model of LonguetHiggins and Lee (1984) fails to give a metrical interpretation for many temporal sequences. The model of Lee

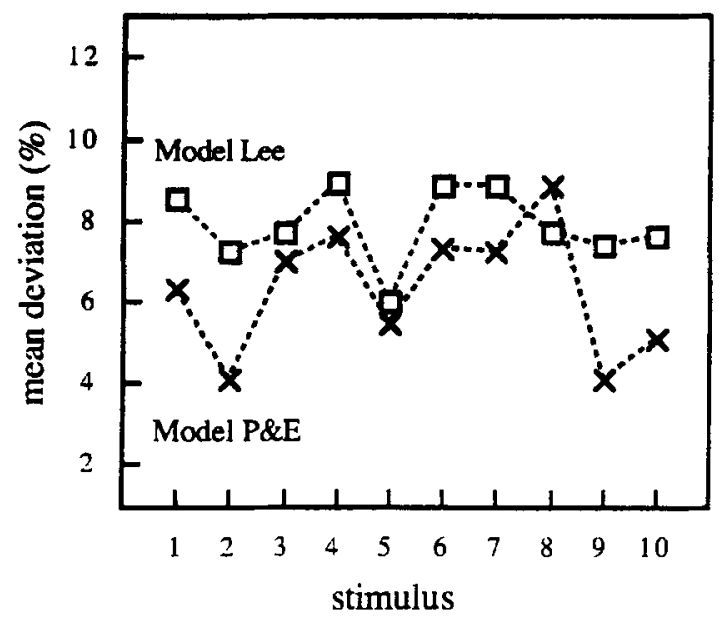

Figure 1. Accuracy of reproduction as a function of metrical structure predicted by the two models, Lee (Lee, 1985) and P\&E (Povel \& Essens, 1985). Lines connect the mean deviations that are in the same condition. 
(1985), which is a refinement of the model of LonguetHiggins and Lee (1982), fares better in this regard and can generate predictions for almost all sequences of the sets used in the analysis. The experimental comparison of predictions by Lee (1985) and Povel and Essens (1985) showed that temporal sequences structured according to the clock model of Povel and Essens are reproduced more accurately than those structured according to Lee's model. From this it can be concluded that the clock model is superior to the model of Lee in predicting the representation for temporal sequences.

Recently, Lee (1991) proposed extensions to his 1985 model. In the 1985 model, the metrical interpretation is built up by combining smaller units into larger units. In the 1991 model, higher order metrical units can be subdivided again into smaller units, thus arriving at a complete metrical description of all intervals in the sequence. This is particularly efficient for the metrical representation of tone sequences that start with relatively large first tone onset intervals. A further extension is the calculation and accumulation of counterevidence in a score similar to the counterevidence score used by Povel and Essens (1985). The revised model does not result in different predictions for the sequences used in our experiments.

The conclusion that the model of Povel and Essens (1985) more accurately predicts the representation for temporal sequences does not discount the idea that early information could influence the process. The experiment was not designed to explore this possibility; it compared the quality of different interpretations imposed on the sequence. Information gathered during the early processing of the sequence can trigger certain hypothetical structures that are subsequently tested by the listener for their adequacy. Moreover, information on structures from former sequences can be carried over. It was observed in some instances that subjects start off with some clock and try to apply it to the sequence presented. Sometimes the adopted clock is fixed so strongly that it is difficult for the subject to switch to a different clock. This is particularly true in respect of clock size. In a pilot experiment in which subjects tapped a beat along with the sequence, a test of switching beat tapping from a 3- to a 4-clock and vice versa revealed that it took on the average four cyclical presentations of the sequence before subjects changed and correctly synchronized the beat tapping again. From these observations, we conclude that humans do not easily change the time unit used for structuring sequences. We do not expect that one interval early in the sequence has a large effect on the likelihood of establishing a new clock.

\section{PART 2 Factors of Complexity}

In the two-stage approach to the coding of temporal sequences, it is implicitly assumed that clock and subdivision characteristics contribute independently to the coding complexity of the sequence. The clock factor refers to the quality of the match of the clock with the sequence, expressed in the C-score. Sequences that have low $\mathrm{C}$-scores are less complex to represent and will be reproduced better than sequences with higher $\mathrm{C}$-scores. Complexity of subdivision derives from the way in which the higher order interval is subdivided. Subdivisions of a clock unit are of three kinds: subdivision into equal parts; unequal subdivision; or empty, meaning that there are no intervals in that clock unit. The examples given in Table 6 illustrate the different subdivision types. The question that we address here is whether unequal subdivisions create extra complexity in the representation of temporal sequences.

Povel and Essens (1985) have argued that unequal subdivisions are more complex because they have to be described by their proportional interval relations, resulting in a less efficient code than that for equal subdivisions, which can be described as a repetition of equal intervals. The fewer symbols that are needed in order to describe the contents of the subdivisions in the sequence, the less complex that sequence is, and the better it can be mentally represented. The examples in Table 6 differ in subdivision complexity: the first example has only one unequal subdivision (subdivision 211), whereas the second has three unequal subdivisions. The conclusion could be that the first sequence ought to be better represented and easier to reproduce than the second. However, there is another factor. As discussed in the first part of this paper, the quality of the match between the best clock and the accents in a sequence (expressed in the Cscore) is also a complexity factor. To test the subdivision hypothesis, both factors - subdivision and clock - have to be separated and varied independently.

Using a two-alternative forced choice paradigm, Povel and Essens (1985) asked subjects to compare stimulus pairs on their complexity (a pair consisted of a test sequence combined with an isochronic sequence inducing either a 4-clock or a 3-clock). The results of this experiment with 12 stimuli supported the complexity predictions of the subdivision model. Recent reanalysis of the experiment, however, revealed a problem of confounding variables. Not only was the number of symbols different between stimuli (as intended), but the match of the clock also differed and, consequently, so did the degree of counterevidence, as defined by the clock model. The data from the complexity judgment experiment of Povel and Essens are summarized in three categories in Table 7: subjects favoring a 3-clock stimulus ("3") as the

Table 6

Types of Subdivision and C-Scores of Two Sample Sequences as a Function of Segmentation by Their Best Clock

\begin{tabular}{|c|c|c|c|}
\hline & Example & Subdivision & $\mathrm{C}^{+}$ \\
\hline 1 & $\begin{array}{l}|||||.||| \ldots ! \\
i \ldots i \ldots i \ldots *\end{array}$ & (1111) (211) (Empty) & 0 \\
\hline 2 & $\begin{array}{l}|\ldots||||.| \mid \ldots ! \\
1 \ldots i \ldots i \ldots *\end{array}$ & $(31) \quad(112)(13)$ & 2 \\
\hline
\end{tabular}

Note-Empty $=$ no subdivision; $(1111)=$ equally subdivided in four; 211,31 , etc. $=$ unequally subdivided. $\quad *$ Predicted 4 -clock. + C-score. 
Table 7

Observed and Predicted Preferred Metrical Structures Based on a 4-Clock or a 3-Clock (Data Adapted From Povel \& Essens, 1985)

\begin{tabular}{llccc}
\hline No. & \multicolumn{1}{c}{ Sequence } & $\begin{array}{c}\text { Subjects } \\
\text { Favor }\end{array}$ & $\begin{array}{c}\text { Subdivision Model } \\
\text { Favors }\end{array}$ & $\begin{array}{c}\text { Clock Model } \\
\text { Favors }\end{array}$ \\
\hline 1 & 31221111 & 4 & 4 & 4 \\
2 & 1111221111 & 4 & 4 & 4 \\
3 & 111122112 & 4 & 4 & 4 \\
4 & 2112213 & $3 / 4$ & $4^{*}$ & $3 / 4$ \\
5 & 312213 & $3 / 4$ & $3 / 4$ & $3 / 4$ \\
6 & 211112112 & $3 / 4$ & $3 / 4$ & $4^{*}$ \\
7 & 11111111121 & 3 & $3 / 4^{*}$ & $3 / 4^{*}$ \\
8 & 3121113 & $3 / 4$ & $3^{*}$ & $3 / 4$ \\
9 & 111111113 & 3 & 3 & $3 / 4^{*}$ \\
10 & 3111113 & 3 & 3 & 3 \\
11 & 11111213 & 3 & 3 & 3 \\
12 & 3111213 & 3 & 3 & 3 \\
\hline
\end{tabular}

Note-3/4 = no clear preference for either the 3-or the 4-clock on the basis of metrical structure. *Predictions that deviate from subjects' judgment.

simpler; favoring a 4-clock stimulus ("4"); and mixed result (" $3 / 4$ "). The predicted preferences based on the subdivision model and the clock model (see Table 7) indicate that either model can predict the data equally well. From this analysis it can be concluded that the test's results were not conclusively in support of the subdivision model.

We assessed the contribution of each factor-clock match and subdivision - to the perceived complexity in temporal sequences by using three paradigms: immediate and delayed reproduction (Experiment 2), and complexity judgment (Experiment 3 ). In immediate reproduction, the subject had to reproduce the sequence immediately after listening. Then, following a time interval during which an interference task was executed to inhibit mental rehearsal, a second reproduction was carried out by the subject. This was termed delayed reproduction. It was anticipated that sequences that were designated as being more complex to represent, either because of subdivision or clock match complexity, would result in higher deviations and errors. In the complexity judgment experiment, the subject judged how simple or complex a stimulus was on a scale.

\section{EXPERIMENT 2}

\section{Method}

Subjects. Six subjects- 4 university students and 2 members of the TNO Human Factors Research Institute-participated in the experiment. Two of the subjects had been trained in playing an instrument for at least 5 years.

Stimuli. From a set of tone sequences of length 16, Set 16, subsets were created with sequences differing in $C$-score $(C=1,3)$ and numbers of complex subdivisions $(4,3$, or 2$)$. This resulted in six sets corresponding to three levels of the complex subdivision condition crossed with two levels of the C-score condition, as presented in Table 8 . For example, tone sequence 8 structured with a 4-clock has a $\mathrm{C}$-score of 1 ; one clock beat falls on a nonaccented tone (which is the 10 th tone). It has three complex subdivisions and one simple subdivision: (112) (1111) (31) (13). Tone Sequence 24 structured with a 4 -clock has a $\mathrm{C}$-score of 3 ; three clock beats fall on a nonaccented tone (the $1 \mathrm{st}, 4 \mathrm{th}$, and 10 th tones). Tone Sequence 24 has two complex and two simple subdivisions: (121) (1111) (22) (13).

The stimuli were presented to the subjects as sequences consisting of tones of $50-\mathrm{msec} 830-\mathrm{Hz}$ square waves, with $3-\mathrm{msec}$ rise and fall times in combination with a low-pitch $(125-\mathrm{Hz}$, square waves) isochronic tone sequence that corresponded to the predicted clock. The sequences were presented cyclically (with no breaks between cycles, as the "!" in Table 8 indicates), but only four times. Stimulus generation and response registration was the same as in Experiment 1.

Procedure. Subjects had to perform three tasks in a row: immediate reproduction; an interference task, which consisted of "counting-backwards-by-three"; and delayed reproduction. Before the experiment started, procedures were practiced with three training stimuli. In the immediate reproduction task, subjects listened to the stimulus and practiced tapping in synchrony. The subject stopped stimulus presentation by pushing a button and then reproduced four cycles of the sequence on a response plate, as precisely as possible. Each tap produced the same tone as that used in the stimulus. If the subject made a serious tapping error in immediate reproduction resulting in a standard deviation that exceeded $15 \%$

Table 8

24 Tone Sequences of Experiments 2 and 3 in the Three Levels of the Subdivision Condition Having Two Levels of Clock Condition (C-Score)

\begin{tabular}{|c|c|c|c|c|}
\hline \multirow{2}{*}{$\begin{array}{c}\text { Complex } \\
\text { Subdivision } \\
\end{array}$} & \multicolumn{4}{|c|}{ C-score } \\
\hline & No. & $\mathrm{C}=1$ & No. & $C=3$ \\
\hline \multirow[t]{4}{*}{ Four } & 1 & $|1| .|||| .1|| .1 \ldots !$ & 13 & $|\ldots|||||,|\ldots| \mid \ldots !$ \\
\hline & 2 & ||$|| ..|1| .|||| \ldots !$ & 14 & $1.1111 .111 .111 \ldots !$ \\
\hline & 3 & $||||. .|||| \ldots|| \mid \ldots !$ & 15 & $1 \ldots 11|| .1|| 1 \mid. \ldots !$ \\
\hline & 4 & $|| .1|\ldots| 1|.1| 1 \mid \ldots !$ & 16 & $|\ldots||| .|1||| \mid. \ldots !$ \\
\hline \multirow[t]{4}{*}{ Three } & 5 & $111.111 .11 .11 \ldots !$ & 17 & $1|.1| 1.1111 .1 \ldots !$ \\
\hline & 6 & $|1| .1 \ldots \mid 1.1 .11 \ldots !$ & 18 & $1 \ldots 1111111.111 \ldots !$ \\
\hline & 7 & 1111111.111 .111 .1 & 19 & $\mid .1 .11 .111 .111 \ldots !$ \\
\hline & 8 & ||$||||||. \ldots|| \mid \ldots !$ & 20 & $11.11|1.1| .11 \ldots !$ \\
\hline \multirow[t]{5}{*}{ Two } & 9 & $|1| 1|| .+1.1|| \ldots !$ & 21 & $|1| .1|| 1||||. \ldots !$ \\
\hline & 10 & $|| ..|.| .|||| \mid. \ldots !$ & 22 & ||$\ldots|| .|||||| \ldots !$ \\
\hline & 11 & $|1| 1|| 1 .|| 1 .|.| . \mid$ & 23 & $|.| .|1.11| 1|1| 1 \ldots !$ \\
\hline & 12 & $|1| .|1 \ldots| .|.| \ldots !$ & 24 & ||$.|||||1.| . \mid 1 \ldots !$ \\
\hline & * & $i \ldots i \ldots i \ldots i \ldots$ & * & $i \ldots i \ldots i \ldots i \ldots$ \\
\hline
\end{tabular}

Note Smallest tone onset interval is $200 \mathrm{msec}$. ${ }^{*}$ For all the 24 sequences, the predicted mental clock (4-clock). 
of the mean of the tapped intervals, the subject was required to repeat the procedure of listening and reproducing. After successfully completing reproduction, subjects received a card with a number which they used as start number for counting backwards by threes. After 10 counts (lasting about $20 \mathrm{sec}$ ), the subjects were stopped and were required to reproduce the sequence a second time for four consecutive cycles, as precisely as possible. The subjects were encouraged to begin and continue tapping even if they were certain that their responses were wrong or when errors occurred during reproduction.

\section{Results}

Immediate reproduction. Mean deviations in the reproductions were used as a measure of the accuracy of reproduction, as in Experiment 1. Examination of the performance of the individuals yielded no differences related to musical training. The results presented in Figure 2 (top panel) are averaged over subjects for the 24 stimuli. The stimuli have been grouped by the two clock conditions (C-score) and the three complex subdivision conditions. Systematic performance differences were found for $\mathrm{C}$-score, with performance significantly less accurate in $\mathrm{C}=3$ than in $\mathrm{C}=1[F(1,5)=11.5, p<$ $.005]$. The data show no significant differences in performance on the basis of the predicted subdivision complexity. Complex subdivision and $\mathrm{C}$-score interaction was not significant. Although there were some clear outliers, overall differences between stimuli were not significant.

Delayed reproduction. The subjects considered this part of Experiment 2 to be very difficult, demanding high concentration. They made many errors, often switching during tapping to a sequence different from the one they started with. Most errors consisted of inserting or deleting tones in the sequence (see Table 9), which resulted

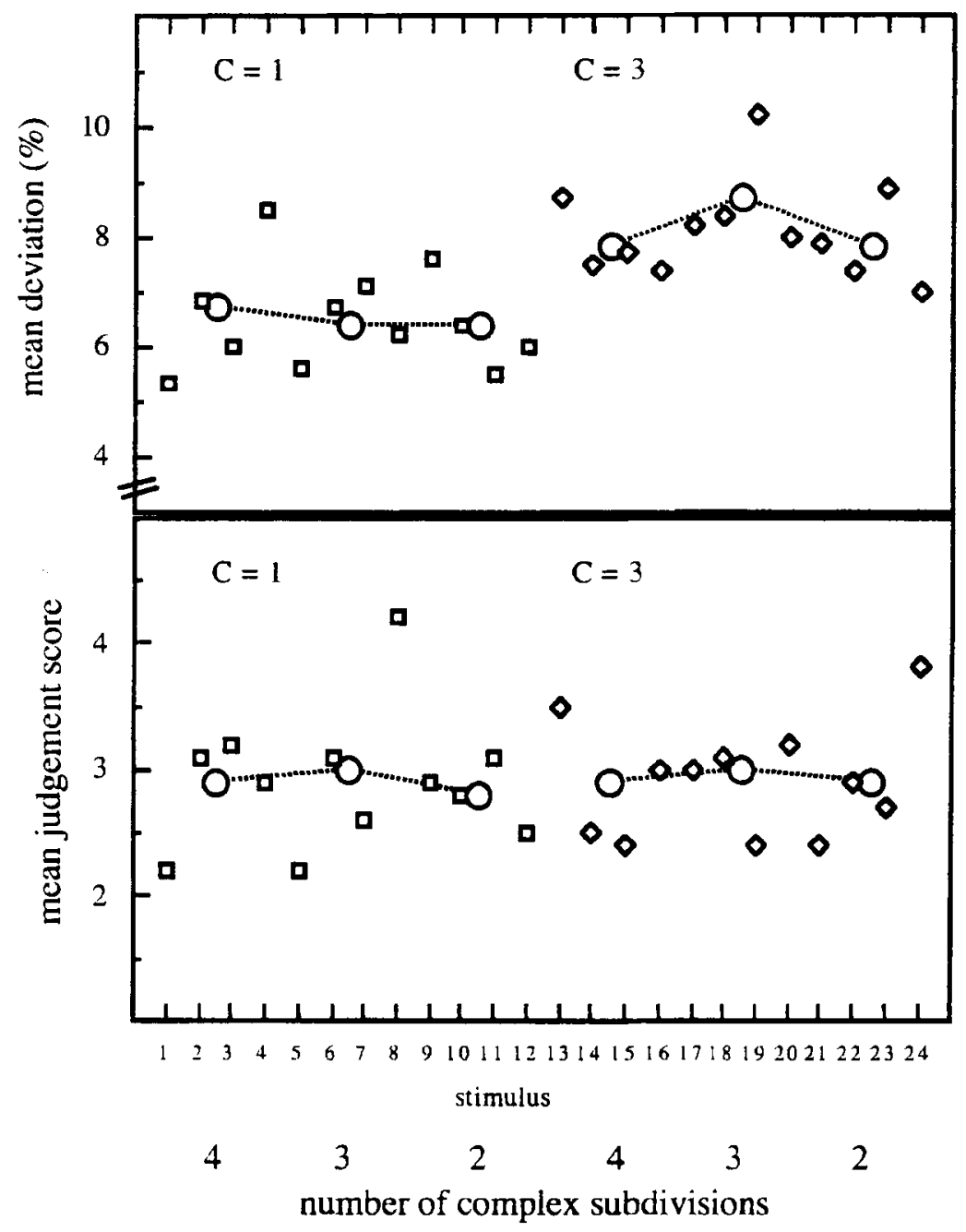

Figure 2. Top panel: accuracy of reproduction in Experiment 2 (immediate reproduction). Bottom panel: complexity judgments in Experiment 3. Data as a function of quality of clock match ( $C$-score; 1 = best match) and subdivision complexity ( 2 = least complex). Dotted lines connect the averages of each complex subdivision level for the two $C$-score conditions (Stimuli 1-12 and 13-24, respectively). 
Table 9

Categories of Errors Observed in Delayed Reproduction

\begin{tabular}{|c|c|c|}
\hline Categories & $N$ & Examples \\
\hline $\begin{array}{l}\text { 1. One or two tones omitted or added } \\
\text { (the same 4-clock still fits) }\end{array}$ & 21 & 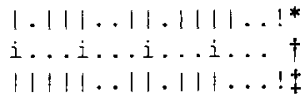 \\
\hline $\begin{array}{l}\text { 2. Large change in tone element structure } \\
\text { (the same 4-clock still fits) }\end{array}$ & 10 & $\begin{array}{l}|1| \cdot|\ldots||\cdot| \cdot \mid 1 \ldots ! \\
i \ldots i \ldots i \ldots i \ldots \\
|.| 1|| .|1 \ldots| \mid \ldots !\end{array}$ \\
\hline $\begin{array}{l}\text { 3. Changed tone element structure } \\
\text { (a 4-clock does not fit or is costly; } \\
\text { C-score }>4 \text { ) }\end{array}$ & 11 & $\begin{array}{l}|1,| 1|1 .| 1 . \mid 1 \ldots ! \\
i \ldots i \ldots i \ldots i \ldots \\
|1| .|1| . \mid 1 \ldots \ldots !\end{array}$ \\
\hline $\begin{array}{l}\text { 4. Correct tone element structure; timing is } \\
\text { changed, making long intervals longer or } \\
\text { shorter }\end{array}$ & 13 & $\begin{array}{l}|1.11| 1 .|1.1| \ldots ! \\
i \ldots i \ldots i \ldots i \ldots \\
11 \ldots 1|1| .|1 .| 1 \ldots !\end{array}$ \\
\hline $\begin{array}{l}\text { Remainder } \\
\text { no stable reproduction; no } \\
\text { interpretation possible }\end{array}$ & 6 & \\
\hline
\end{tabular}

in high deviation scores, because timing was measured against the given sequence. An error reproduction was defined as one that had a deviation score of greater than $15 \%$. The score reflects the deviation of the reproduced from the given sequence. Large deviation scores are a result of changes in the representation of a sequence. Smaller deviations reflect timing errors without a change in the structure of the sequence. A breakpoint of $15 \%$ was judged to be adequate for identifying all the structural changes. The total number of errors was 61 (out of 144 observations). For the $C=1$ and $C=3$ conditions, the percentages of errors were 13,20 , and 8 , and 13,25 , and 21 , for four, three, and two complex subdivisions, respectively. Most of the errors were found in the $C=3$ condition. In a log-linear analysis (Wilkinson, 1988) of the distribution of errors no significant effects or interactions were found in the data.

In the debriefing session, 4 subjects referred to specific strategies for capturing the sequence. They perceived the tones in the sequence as forming clusters and treated these clusters and the "rhythm" as separate but linked characteristics of the sequence. Their strategy was to first pick up the time structure of the sequence and then memorize the size of the tone clusters, usually as numbers. The two characteristics were combined during reproduction, resulting in a positioning of the clusters against the rhythm. This strategy might correspond to the distinction made by Bamberger (1978) between figural and durational coding, with figural coding being the coding of the sequence in terms of tone clusters, and the durational coding, the timing in the sequence.

The reproductions were further analyzed to see whether the strategies used by the subjects led to errors that were related to the figural structure of the sequence. To find the figural structure of the reproduced sequences, we filtered the tapped intervals, rounding the intervals of the reproduced sequence to the nearest valid interval value $(200,400,600,800,1,000$, longer $)$. For instance, the application of the rounding rule to the sequence $228,190,440,570,205,842$ results in 200,200 , $400,600,200,800$ (or 112314). In some reproductions, erroneous tapping occurred after a correct start; in others, reproduction started incorrectly, but was later corrected, suggesting that the subject remembered the sequence while tapping. Categorization of the errors was done only for those erroneous reproductions in which at least two consecutive cycles had the same type of error. Otherwise that reproduction was categorized as "Remainder." The nature of the errors suggested four categories as defined in Table 9. The first three indicate changes in figural structure having varying consequences for the time structure; the fourth category reflects pure timing errors where the correct figural structure is maintained. Assignment to these categories was straightforward and was based on the comparison between given and reproduced sequence.

Categories 1 and 2 differ in the amount of similarity between the presented and reproduced sequences. In Category 1 , the reproductions differ by only one or two tones from the original. Changes result from adding or deleting a tone, or a combination of both. Errors in Category 2 reflect large structural changes in the sequence as reproduced. Despite the structural changes in Categories 1 and 2, a 4-clock still fits the sequence. In terms of clock complexity, the reproduced sequences have equal or less complexity than the original sequence. In Category 3 , the large change in element structure results in a sequence having no clock that fits or only a costly one $(C>4)$. Most of the Category 4 errors (11 out of 13) occurred in the $\mathrm{C}=3$ condition. Here the exact tone element structure was reproduced, but the duration of the long intervals between the tone clusters was changed. Seven of these error reproductions had an improved C-score in comparison with that for the given sequence. 


\section{EXPERIMENT 3}

In Experiment 3, clock and subdivision factors were further assessed by using human listeners' complexity judgments. Both complexity against the clock $(\mathrm{C}=1$ and $\mathrm{C}=3$ ) and the subdivision (number of complex subdivisions: four, three, and two, in decreasing complexity) were varied.

\section{Method}

Subjects. Twenty subjects, members of the TNO Human Factors Research Institute, participated in the experiment. Five had been trained in playing an instrument for at least 5 years. Two subjects who participated in both Experiments 2 and 3 did Experiment 3 first.

Stimuli. The stimuli were the same as in Experiment 2.

Procedure. The task of the subjects was to judge the complexity of the stimulus on a 5-point scale. The simple-complex dimension was represented on the top of the answer sheet as (translated from Dutch) very simple, moderately simple, neutral, moderately complex, very complex. For each stimulus, there was a horizontal line with five points, matching the simple-complex dimension on the top of the sheet. Subjects marked their choice for each stimulus by crossing a point on the scale. The subjects did not receive any instruction about what was meant by simple or complex. They practiced the task in six trials with stimuli similar to the ones in the experimental sets. The order of presentation of the stimuli was randomized. The subjects listened to each stimulus and marked their judgments on the answer sheet. The subjects were given the option to indicate on a time line with beginningmiddle-end positioned to the right of each scale whether the perceived complexity was at a specific location in the sequence.

\section{Results}

The distribution of frequencies for the judgment scores on the 1-5 simple-complex scale comprised 52 , $126,145,127$, and 30 , respectively. The results are presented in Figure 2 (bottom panel) for the three complex subdivision conditions in the two C-core conditions. No significant effects were found for the predicted complexity differences in the complex subdivision condition. No effect was found for C-score, either. However, stimuli did differ significantly from each other $[F(18,342)$ $=6.9, p<.001]$. The three stimuli considered as most complex were Nos. 13, 24, and 8 (in ascending order); at the other end of the scale, as least complex, were Sequences 15, 5, and 1 (in descending order). No higher order interactions were significant.

In order to assess other possible factors that might contribute to the simple-complex dimension, the effect of stimuli was further analyzed and related to the results of delayed reproduction. Stimuli were ranked on their judgment scores and matched to the error data of Experiment 2. Sequences ranked in the upper half of the judgment scores contained about twice as many errors as those in the lower half. The rank-order correlation for the judgment scores and number of reproduction errors was positive and significant $\left[r_{\mathrm{s}}(24)=.51, p<.01\right]$.

The subjects did not, in general, use the opportunity to indicate at which point in the sequence a specific complexity was heard. In the debriefing session, 7 subjects mentioned ease of reproduction as a criterion for judgment. (Subjects are usually aware of the beginning point of a cyclically presented sequence.) Three subjects mentioned that sequences for which they perceived no clear beginning in the cyclical presentations were considered as complex. Sequence 11 (see Table 8) was mentioned as an example.

\section{Discussion}

Two potential factors contributing to complexity in the coding of temporal sequences were assessed. One was the degree of match between the mental clock and the perceived accents in a sequence. The complexity predictions of the clock model were confirmed in the reproduction task: sequences with low C-scores resulted in a more accurate representation than did those with high $\mathrm{C}$-scores. In the judgment task, however, the differences in clock match did not correspond to the differences in judged complexity. The latter results suggest that another factor is at play when subjects are asked to judge complexity. This will be discussed below. The second potential factor of complexity was the nature of the subdivisions of the higher order clock units. Povel and Essens (1985) had argued that the more unequal subdivisions a sequence has, the more complex it is. However, neither the results from the reproduction nor the judgment supported the subdivision complexity hypothesis. From these findings, it can be concluded that the nature of the subdivision is not a determinant of coding complexity. The judgment results differ from the results of Povel and Essens (1985), and this difference remains to be explained.

The errors that people make in retaining temporal sequences give insight into the way the sequences are represented. Most of the errors were changes in the tone element structure of the sequence, resulting from omitting or adding tones. However, the original clock was preserved. Certain tones in the sequence seem to have been more critical for the representation than others-in particular, those that were connected to the beat. They are less likely candidates for errors, because omission of such tones would negatively affect the overall quality of the clock. The addition and deletion of tones may change the position of the accents and thus change the quality of the match of the clock, in a positive or a negative sense. For instance, if the beat coincides with the second tone of a two-tone cluster, the addition of a third tone at the end of the cluster shifts the accent away from the beat, resulting in a poorer match. Most error reproductions had a clock match that was equal to or better than the original sequence. For example, it was found that in reproduction the sequence with intervals of 400,200 , $200,600,200,400,200,200,200,600 \mathrm{msec}$ was changed: a tone was added in the first interval, thus splitting it into $200,200 \mathrm{msec}$. The last two tone intervals, 200 and 600 , were merged into $800 \mathrm{msec}$ as a result of the deletion of the tone that separated them. The 4-clock with a size of $800 \mathrm{msec}$ still fit the reproduced sequence, but the match was improved because deleting the last tone brought an accent to the beat. The error data 
suggest a representation of a sequence that consists of a temporal hierarchy that defines where tones can be positioned in time. The beat forms the core of the hierarchy and anchors the tone sequence against the temporal hierarchy.

The judgment data did not show an effect of clock match (C-score), whereas the reproduction data did. One possible explanation is that $\mathrm{C}$-scores may be more applicable when precise timing is involved; another is that the difference between the $\mathrm{C}$-scores was not large enough to affect the complexity judgments. Apparently the judgments tapped other information from the sequence than the match of clock and perceived accents. It is known that judgments of complexity are multidimensional in nature (Gabrielsson, 1973). The data were further analyzed to identify systematic factors that could account for the differences in complexity judgments. In particular, we looked at regularities in the sequence. The judgments were ranked and the sequences at the two ends of the ranked data were compared. The difference between those sequences suggests that certain recurring relationships between beat and tone cluster may have been used by the subjects as a basis for judging whether a sequence was simple or complex. For instance, in the sequence that was judged as the simplest (Sequence 1; see Table 8), all tone clusters (tone groups consisting of two or more tones) had their first tone on the beat. In contrast, in sequences that were judged as complex (e.g., Sequence 24), there were various relationships between the tone clusters and beat.

The beat gives stress to the tone with which it coincides and thus marks upbeat and downbeat tones in the tone cluster. If we characterize a tone-cluster-beat relationship as a signal envelope, with the beat as the signal and the tone cluster coinciding with the beat forming the envelope of that beat, three patterns can be defined: (1) one or more tones preceding the beat - a "rise pattern"; (2) one or more tones following the beat-a "fall pattern"; and (3) one tone or a tone cluster marked by the beat(s)-a "step pattern." A signal envelope can consist of a single one of these patterns or a combination. Large tone clusters form envelopes that can consist of two or more beats. With more than two beats involved, the inside beat(s) is (are) considered to have no outstanding pattern and the beats at the beginning and end are treated as separate envelopes that can differ in pattern.

Variations in tone-cluster-beat relationship within a tone sequence have the effect of violating the envelope expectations built up by the subject during the processing of the sequence and may be perceived as increasing complexity. The first envelope of a sequence sets the expectation. A variation is perceived if the current envelope is different from the preceding envelope. The amount of variation is given by the number of patterns in the current envelope that are new in comparison with the pattern(s) in the previous envelope. Summing the variations in a sequence gives an envelope variation score (EV) of that sequence. For example, consider Stimulus 24 from Table 8 . It has three tone-cluster-beat en- velopes; the second and third beat are grouped in one envelope by the tone cluster. The patterns in the envelopes are: "fall," "rise and step," and "fall." There are three variations in the succession of envelopes. Both the rise and the step of the second envelope differ from the fall pattern in the previous envelope, and the fall pattern in the last envelope differs from the rise-step patterns in the previous envelope $(E V=3)$. Another example is Sequence 15. There, the second envelope has two patterns (rise and fall) that deviate from the first envelope, which has a step pattern. The other envelopes are repetitions of the second, so that the EV score of the sequence is 2. The EV scores for the 24 stimuli of Experiment 3 are given in Table 10.

Evidence for the hypothesis that the successive variation in tone-cluster-beat relationship is a factor in the perceived complexity of a tone sequence was sought by analyzing the data of Experiment 3. The EV algorithm was applied to the 24 stimuli and the resulting EV scores were correlated to the judgment data (Table 10). A significant positive relationship was found $\left[r_{\mathrm{s}}(24)=.71\right.$, $p<.01]$, indicating that this factor gives a partial description of the simple-complex dimension in the judgment data. It can be concluded that the changes in the relationship between beats and tone clusters is a factor contributing to the complexity perceived in tone sequences.

\section{GENERAL DISCUSSION}

Four algorithmic models from the literature on the perception of temporal sequences have been compared. They differ in terms of which information from the tone sequence is considered critical and in how that information is integrated to form a metrical interpretation of the sequence. When the algorithms were applied to a large set of sequences, only two of the models provided predictions for most of the sequences (the clock model of Povel \& Essens, 1985, and the model of Lee, 1985). Both models look for markers of higher order time intervals to structure a tone sequence. Both identify as

Table 10

Envelope Variation Scores (EV) for the Stimulus Set of Experiment 3 and Corresponding Average Judged Complexity

\begin{tabular}{rccccc}
\hline No. & $\begin{array}{c}\text { Judged } \\
\text { Complexity }\end{array}$ & EV & No. & $\begin{array}{c}\text { Judged } \\
\text { Complexity }\end{array}$ & EV \\
\cline { 1 - 5 } 1 & 2.2 & 0 & 13 & 3.5 & 2 \\
2 & 3.1 & 3 & 14 & 2.5 & 2 \\
3 & 3.2 & 2 & 15 & 2.4 & 2 \\
4 & 2.9 & 2 & 16 & 3 & 2 \\
5 & 2.2 & 1 & 17 & 3 & 2 \\
6 & 3.1 & 3 & 18 & 3.1 & 2 \\
7 & 2.6 & 0 & 19 & 2.4 & 2 \\
8 & 4.2 & 4 & 20 & 3.2 & 2 \\
9 & 2.9 & 1 & 21 & 2.4 & 2 \\
10 & 2.8 & 1 & 22 & 2.9 & 2 \\
11 & 3.1 & 1 & 23 & 2.7 & 2 \\
12 & 2.5 & 1 & 24 & 3.8 & 3 \\
\hline
\end{tabular}

Note-The complexity scale ranges from 1 (simple) to 5 (complex). 
markers the tones that onset the relatively long intervals ("accents"). In addition, the clock model uses the tone that ends a relatively long interval as a marker. The models are comparable in terms of the information used. The models diverge in the ways in which the metrical interval is established and in how this higher level interval fits the sequence. Lee's model determines the first marker-bounded interval and tests whether this is an adequate metrical interval. The clock model attempts to fit equal-interval time scales (clocks) to the accents in the whole sequence and looks for a clock that minimizes the cost when a match is not perfect. As a result, the selection of the clock is not governed by a specific order of tone intervals. The clock model is therefore sensitive to information in the whole sequence. The experimental comparison between the two models shows that the clock model is a better predictor of the metrical structure of a tone sequence than is Lee's model.

The clock model seems to capture critical aspects of the complexity of temporal sequences; the quality of the fit is indicative of the quality of the reproduction. The validity of the clock model is supported by many experiments. It is robust in the sense of generating the same results in reproduction tasks performed in different contexts with different subjects using different sequences. The quality of performance, in terms of a reproduction deviation score, is comparable over experiments: $6 \%-8 \%$.

The other proposed account of the complexity of temporal sequences, formulated as the subdivision model, did not fare well in our experiments. The data showed that subdivision complexity had no effect on the accuracy of the representation and was not a factor in judgment of the complexity of the sequence. In other words, the way the clock unit is subdivided does not account for the complexity of the sequence. This raises the question of whether subdivision is a component of the coding process.

Neither the clock nor the nature of the subdivisions accounts for the results from the complexity judgment experiment. Instead, the results can be explained by the relationship of the tone clusters in the sequence to the beats of the clock. The tone that coincides with the beat is the prominent tone of the cluster; the other tones are represented as the envelope around it. We formulated the envelope variation model, which states that the variation in the envelopes is a factor in the perceived complexity of a tone sequence. This account of the judgment data is based not on the temporal characteristics of the sequence but on the interplay between the tone clusters and the clock.

The errors that subjects made in delayed reproduction of the sequences also suggest an interplay between the temporal and figural structures of a sequence. The errors were described as changes in the figural structure resulting from the addition or deletion of tones. Although these modifications change the interval structure of the sequence itself, the overall relationship between the clock and the sequence did not seem to be affected. More specifically, when the original sequence had inter- vals fitting into a 4-clock, the reproduced version did as well and only those tones that were not connected to the beats of the clock were candidates for change.

The results from the reproduction and judgment experiments and from the error observations suggest a dual-code representation of tone sequences that comprises temporal and figural characteristics. The temporal part of the code contains information about the metric of the clock level and lower levels. If a tone is added in error, the tone will be timed so that it fits into the current temporal metric. The figural part of the code contains information about the configuration of the tone clusters in relation to the beat.

The two-stage account of the perception of temporal sequences given by Povel and Essens (1985) should be modified. Specification of the intervals in a sequence in terms of subdivisions is not needed. The dual-code model that we propose here incorporates and extends the clock model of Povel and Essens. The clock model as originally formulated specified which clock matches best with the accents perceived in the sequence. It did not, however, specify the metric of the levels below the beat. More specifically, it did not specify the possible timings of the tones in the sequence. Essens (1986) concluded that there are basically two metrics for the levels below the clock, a division of the clock unit into 3 or 4 (or 2 and 2) equal time intervals. These correspond to schemes commonly used in Western music (LonguetHiggins, 1976). Jones and Yee's (1993) concept of hierarchical time ratios abstracted by listeners from temporal sequences is analogous to this account. It is proposed now that establishing a clock implies the use of either a 3-metric or a 4-metric. Thus, the temporal code specifies the metric of the sequence. The figural part of the code captures the location of the tones at the lower level. It specifies how the tone clusters are anchored by the beat. As suggested by the envelope variation model, tone clusters can consist of tones preceding the beat, on the beat, or after the beat.The figural code is shaped by the clock. If no clock is available, there is only a sequence of tone clusters with a timing between tones governed by a simple 2:1 scheme (Fraisse, 1946). Although the dualcode proposal is less succinct than a purely temporal account, it seems to be more realistic, in that it integrates both aspects of tone sequences-temporal and figural.

\section{REFERENCES}

Bamberger, J. (1978). Intuitive and formal musical knowing: Parables of cognitive dissonance. In S. S. Madeja (Ed.), The arts, cognition and basic skils (pp. 173-206). New Brunswick, NJ: Transactions Books.

EsSENS, P. J. (1986). Hierarchical organization of temporal patterns. Perception \& Psychophysics, 40, 69-73.

Essens, P. J., \& Povel, D.-J. (1985). Metrical and nonmetrical representations of temporal patterns. Perception \& Psychophysics, 37, $1-7$.

Fraisse, P. (1946). Contribution à l'étude du rythme en tant que forme temporelle. Journal de Psychologie Normale et Pathologique. 39. 283-304.

Fraisse, P. (1956). Les structures rythmiques. Louvain: Publications Universitaires de Louvain. 
GABRIELSSON, A. (1973). Similarity ratings and dimension analyses of auditory rhythm patterns. Scandinavian Journal of Psychology, 14, 138-160.

JONES, M. R. (1976). Time, our lost dimension: Toward a new theory of perception, attention, and memory. Psychological Review, 83, 323-355.

JONES, M. R., \& YEE, W. (1993). Attending to auditory events. In S. McAdams \& E. Bigand (Eds.), Thinking in sound: The cognitive psychology of human audition (pp. 69-112). Oxford: Oxford University Press

LEE, C. S. (1985). The rhythmic interpretation of simple musical sequences: Towards a perceptual model. In P. Howell, I. Cross, \& R. West (Eds.), Musical structure and cognition (pp. 53-69). London: Academic Press.

LEE, C. S. (1991). The perception of metrical structure: Experimental evidence and a model. In P. Howell, R. West, \& I. Cross (Eds.), Representing musical structure (pp. 59-127). London: Academic Press.

Longuet-Higgins, H. C. (1976). Perception of melodies. Nature, 263, 646-653.

Longuet-Higgins, H. C., \& LeE, C. S. (1982). The perception of musical rhythms. Perception, 11, 115-128.

Longuet-Higgins, H. C., \& LeE, C. S. (1984). The rhythmic interpretation of monophonic music. Music Perception, 1, 424-441.

MARTIN, J. G. (1972). Rhythmic (hierarchical) versus serial structure in speech and other behavior. Psychological Review, 79, 487-509.

Monahan, C. B., Kendall, R. A., \& Carterette, E. C. (1987). The effect of melodic and temporal contour on recognition memory for pitch change. Perception \& Psychophysics, 41, 576-600.

POVEL, D.-J. (1981). Internal representation of simple temporal patterns. Journal of Experimental Psychology: Human Perception \& Performance, 7, 3-18.
Povel, D.-J., \& Essens, P. J. (1985). Perception of temporal patterns. Music Perception, 2, $411-440$.

Povel, D.-J., \& OKKerman, H. (1981). Accents in equitone sequences. Perception \& Psychophysics, 30, 565-572.

WILKINSON, L. (1988). SYSTAT: The system for statistics. Evanston, IL: SYSTAT, Inc.

\section{NOTES}

1. Povel and Essens (1985) used the term "coding model," which might be confusing in that it suggests that only the second stage deals with coding of the sequence. In this paper, coding refers to the structural breakdown of a sequence that results in a representation of the sequence.

2. The results of Povel and Essens (1985) indicate that sequences with one $-\mathrm{ev}$ result in the same reproduction performance as do sequences with two and three 0ev's. The exact relationship not being known, $W$ is best set to an (arbitrary) value larger than the maximum number of 0ev's in the sequences used. For the set of sequences used here of unit lengths 12 and $16, \mathrm{~W}$ is set to 4 (as used by Povel \& Essens, 1985).

3. Longuet-Higgins and Lee use the term "note" to mean the onset of an interval; a long note is thus a tone followed by a long interval (Lee, 1985, p. 57; Longuet-Higgins \& Lee, 1984, p. 431). Although they refer to "musical sequence," the sequences discussed comprise notes that do not differ with respect to pitch, harmony, loudness, or other note characteristics (Lee, 1985, p. 53; Longuet-Higgins \& Lee, 1984, p. 424), and therefore are pure temporal sequences, as used here.

(Manuscript received December 14, 1992; revision accepted for publication October 11,1994 .) 